\title{
Synthetic and Antimicrobial Studies of Some New Chalcones of 3-Bromo-4-(p-tolyl sulphonamido) acetophenone
}

\author{
MISS RASHMI JAIN, O.P. CHOURASIA* and J. TIRUMALA RAO \\ Department of Chemistry \\ Dr. H.S. Gour Vishwavidyalaya, Sagar (M.P.) 470003
}

Received 22 March 2004; Accepted 1 May 2004

\begin{abstract}
Eleven new chalcones have been sysnthesised by condensing 3bromo-4-(p-tolyl sulphonamido) acetophenone with different aromatic aldehydes using the method or Rohrman et al. The antimicrobial activity of these chalcones has been tested by adopting "paper disc diffusion plate method", against various pathogenic fungi(10) and bacteria (9). It has been found that the chalcones have considerable antifungal activity but less antibacterial activity. The results show that these chalcones may find use as antifungal agents.
\end{abstract}

Key words: Chalcones, Antomicrobial study.

\section{Introduction}

Sulphonamides are well known for their therapeutic activity ${ }^{1}$, chalcone derivatives also possess anthelmintic ${ }^{2}$, germicidal ${ }^{3}$. Antimicobial ${ }^{4-5}$ and carcenogenic ${ }^{6}$ activities. It was throught worthwhile to synthesise some bromo sulphonamido chalcones and study their antimicrobial activity against various fungi and bacteria.

Eleven bromo sulphonamideo chalcones have been prepared by condensing 3-bromo-4-(p-tolylsulphonamido) acetophenone with different aromatic aldehydes adopting the method. ${ }^{7}$

\section{Experimental}

In the preparation of 3-bromo-4-(p-tolyl sulphonamideo) acetophenones, two steps are involved.

Preparation of 3-bromo-4-(p-tolyl sulphonamido) acetophenone :

It is also accomplished into two steps:

(a) Synthesis of 3-bromo-4-amino acetophenone - It was prepared by acetylation of acetanilide by "Friedel-Craft's reaction" and the acetylated product then subjected to bromination to get bromo-aceto acetanilide. To separate the side chain acetanilide dissolved in pyridine and residue was taken and hydrolysed in presence of alcoholic $\mathrm{KOH}$ solution to get 3-bromo-4-amino acetophenone.

(b) Condensation of 3-bromo-4-amino acetophenone with p-tolyl sulphonyl chloride - Solution of 3bromo-4-amino acetophenone and p-tolyl sulphonyl chloride in benzene were mixed together and concentrated on water bath. The product was crystallised from ethanol. 
Preparation of 3'-bromo-4-(p-tolyl sulphonamido) chalcone To a solution of 3-bromo-4-(p-tolyl sulphonamido) acetophenone $(0.01 \mathrm{~mole})$ and an aromatic Aldehyde ( 0.01 mole) in minimum quantity of hot aldehyde free alcohol, added $10 \%(10 \mathrm{ml})$ of aq. $\mathrm{NaOH}$. The mixture was stirred for 3 hours at $25-30^{\circ} \mathrm{C}$ and kept at $0^{\circ} \mathrm{C}$ for $24 \mathrm{hrs}$. The product was filtered and recrysytallised from ethanol. Melting points were recorded on Toshniwal melting point apparatus and are tabulated in Table -I.

The chalcones thus prepared were characterised by halochromium test with conc. $\mathrm{H}_{2} \mathrm{SO}_{4}$ and their $\mathrm{IR}^{7}$ and $\mathrm{UV}^{8}$ spectra.

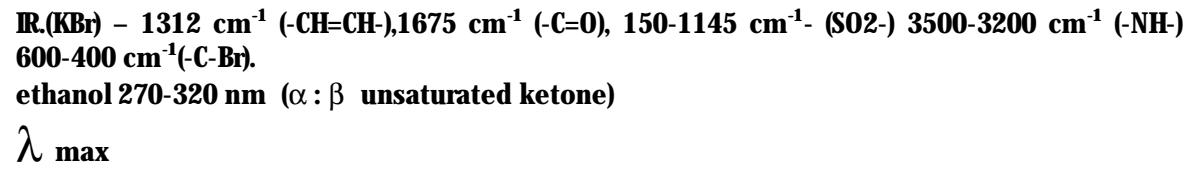

Investigations on the Antimicrobial Activity of the chalcones:

The antimicrobial activity of these chalcones was tested by "paper disc diffusion plate method". For the determination of antifungal activity, about $20 \mathrm{ml}$ of sterilized PDA -(potato-dextrose-agar) and in case of antibacterial screening, oxoid nutrient medium was poured in each sterilised petridish and before gelation of the media, about $2 \mathrm{ml}$ of homogeneous mixture of fungi/bacteria in cool sterilise broth of potato and dextrose/beef extract and peptone respectively was mixed in each petridish. After half an hour, when the media was gelatinized, discs of $6 \mathrm{~mm}$ size prepared from Whatman filter paper (No.1) thoroughly moistened with the solution of chalcone $(4 \%)$ in ethylene glycol were placed over the seeded media and incubated at $32 \pm 7^{\circ} \mathrm{C}$ and $28 \pm 7^{\circ} \mathrm{C}$ for 72 hours in case of fungi and 24 hours in case of bacteria respectively.

Activity of standard antifungal drug, gresiofulvin and antibacterial drug, streptomycin were also checked under the same conditions and concentration. Solvent ethylene glycol also tested for their antimicrobial activity and has shown no activity.

The experiments were performed in duplicate and average zones of inhibition in $\mathrm{mm}$ (including the size of the discs have been recorded and tabulated in Tables II and III ).

\section{Results and Discussion}

On going through the results of antifungal activity (Table II), it has been observed that 3'-bromo-4'-(ptolyl sulphonamido)chalcone and 2-hydorxy-3'-bromo-4'-(p-tolyl sulphonamido) chalcone having very good activity (108.7 and $78.26 \%$ ) when compared with a standard antifungal drug "gresiofulvin" against fusarium moniliform. 2,4-dihydroxy-3'-bromo-4'(p-tolyl sulphonamido) chalcone shows good activity (78.26\% and $65.21 \%$ ) against Gleosporium sp. and Rhizopus stolonifer.

3,4-Dimethoxy,5,3'dibromo-4'(p-tolyl sulphonamido)chalcone shows very good activity $(70.37 \%$, $65.21 \%$ and $66 \%$ ) against Acremonium furcatum, Rhizopus stolonifer and Chaetomium sp.

4-Nitro-3'-bromo-4'(p-tolyl sulphonamido) chalcone inhibits the growth of Gleosporium sp. as equivalent to the standard gresiofulvin and its activity is $70 \%$ against Chaetomium sp.

Remaining chalcones have shown considerable activity against most of the tested fungi; except against Aspergillus niger and Absidium sp. against which all these compounds are inactive.

The results of antibacterial activity has shown that 4-methoxy-3'-bromo-4'(p-tolyl sulphonamido) chalcone have $72.41 \%$ activity against Lactobacillus sp. and activity of 2,4-dihydroxy-3'-bromo-4'-(ptolyl sulphonamido)chalcone against Pseudomonas putida is also good $75.86 \%$ to the standard 'streptomycin'. None of the chalcones have found to be active against Rhizopus lequminosarum, Serratia bachoria and Salmonella typhi (Table III).

On the basis of the results of these antimicrobial efficacies, it can be concluded that these chalcones may find use as antifungal agents. 
Table - I

\begin{tabular}{|c|c|c|c|c|c|c|c|c|}
\hline \multirow[b]{2}{*}{ S.No } & \multirow[b]{2}{*}{ D. Name of the compound } & \multirow[b]{2}{*}{ Molecular formula } & \multirow[b]{2}{*}{ M.pt. $\left({ }^{\circ} \mathrm{C}\right)$} & \multirow{2}{*}{$\begin{array}{l}\text { Yeild } \\
\text { (\%) }\end{array}$} & \multicolumn{2}{|c|}{$\% \mathrm{C}$} & \multicolumn{2}{|c|}{$\% \mathrm{H}$} \\
\hline & & & & & Found & Calcu. & Found & Calcu. \\
\hline 1. & 3'-Bromo-4'-(p-t.s.)C. & $\mathrm{C}_{22} \mathrm{H}_{18} \mathrm{O}_{3} \mathrm{NSBr}$ & $\begin{array}{c}330 \\
\text { (not melted) }\end{array}$ & $60 \%$ & 56.92 & 57.64 & 3.19 & 3.93 \\
\hline 2. & 2-Hydroxy-3'-bromo-4'-(p-t.s.) C. & $\mathrm{C}_{22} \mathrm{H}_{18} \mathrm{O}_{4} \mathrm{NSBr}$ & $260(D)$ & $78 \%$ & 55.01 & 55.69 & 3.53 & 3.79 \\
\hline 3. & 2,4-ihydroxy-3'-bromo-4'-(p-t.s.) C. & $\mathrm{C}_{22} \mathrm{H}_{18} \mathrm{O}_{5} \mathrm{NSBr}$ & 200(D) & $65 \%$ & 52.89 & 53.87 & 3.45 & 3.67 \\
\hline 4. & 2-Methoxy-3'-bromo-4'- (p-t.s.) C & $\mathrm{C}_{23} \mathrm{H}_{20} \mathrm{O}_{4} \mathrm{NSBr}$ & $\begin{array}{c}300 \\
\text { (not melted) }\end{array}$ & $45 \%$ & 55.98 & 56.55 & 3.96 & 4.09 \\
\hline 5. & 4-Methoxy-3'-bromo-4'- (p-t.s.) C & $\mathrm{C}_{23} \mathrm{H}_{20} \mathrm{O}_{4} \mathrm{NSBr}$ & 97 & $60 \%$ & 56.02 & 56.55 & 4.00 & 4.09 \\
\hline 6. & $\begin{array}{l}\text { 3-Methoxy-4-hydroxy-3'- } \\
\text { bromo-C(p-t.s.) C }\end{array}$ & $\mathrm{C}_{23} \mathrm{H}_{20} \mathrm{O}_{5} \mathrm{NSBr}$ & 220 & $75 \%$ & 54.15 & 54.76 & 3.62 & 3.96 \\
\hline 7. & $\begin{array}{l}\text { 3-Methoxy-4-hydroxy-3',5- } \\
\text { dibromo-4'-(p-t.s.) C }\end{array}$ & $\mathrm{C}_{23} \mathrm{H}_{19} \mathrm{O}_{5} \mathrm{NSBr}_{2}$ & 270(D) & $70 \%$ & 47.03 & 47.50 & 3.05 & 3.27 \\
\hline 8. & $\begin{array}{l}\text { 3,4-Dimethoxy-3',5- } \\
\text { dibromo-4'-(p-t.s.)C }\end{array}$ & $\mathrm{C}_{24} \mathrm{H}_{21} \mathrm{O}_{5} \mathrm{NSBr}_{2}$ & 260(D) & $65 \%$ & 47.93 & 48.40 & 3.33 & 3.52 \\
\hline 9. & 3-Nitro-3'-bromo-4'- (p-t.s.) C & $\mathrm{C}_{22} \mathrm{H}_{17} \mathrm{O}_{5} \mathrm{~N}_{2} \mathrm{SBr}$ & 320(D) & $80 \%$ & 52.06 & 52.69 & 3.19 & 3.39 \\
\hline 10. & 4-Nitro-3'-bromo-4'- (p-t.s.) C & $\mathrm{C}_{22} \mathrm{H}_{17} \mathrm{O}_{5} \mathrm{~N}_{2} \mathrm{SBr}$ & 310(D) & $78 \%$ & 51.96 & 52.96 & 3.20 & 3.39 \\
\hline 11. & 4-Chloro-3'-bromo-4'- (p-t.s.) C & $\mathrm{C}_{22} \mathrm{H}_{17} \mathrm{O}_{3} \mathrm{NSClBr}$ & 300(D) & $40 \%$ & 53.52 & 53.82 & 3.16 & 3.46 \\
\hline
\end{tabular}


Table ॥

\begin{tabular}{|c|c|c|c|c|c|c|c|c|c|c|c|}
\hline \multirow{2}{*}{\multicolumn{2}{|c|}{$\begin{array}{l}\text { S.No. Names of the } \\
\text { compound }\end{array}$}} & \multirow[b]{2}{*}{$\begin{array}{l}\text { Fusa rium } \\
\text { monili } \\
\text { form }\end{array}$} & \multicolumn{9}{|c|}{$\begin{array}{l}\text { Zones of inhibition in mm } \\
\text { Name of the organism tested }\end{array}$} \\
\hline & & & $\begin{array}{l}\text { Colleto- } \\
\text { trichum } \\
\text { dematium }\end{array}$ & $\begin{array}{l}\text { Rhizopus } \\
\text { stolonifer }\end{array}$ & $\begin{array}{l}\text { Asper } \\
\text { gillius } \\
\text { niger }\end{array}$ & $\begin{array}{l}\text { Alter- } \\
\text { naria } \\
\text { sp }\end{array}$ & $\begin{array}{l}\text { Acre- } \\
\text { monium } \\
\text { furcatum }\end{array}$ & $\begin{array}{l}\text { Gleos } \\
\text { porium } \\
\text { sp }\end{array}$ & $\begin{array}{l}\text { Chaetomium } \\
\text { sp. }\end{array}$ & $\begin{array}{l}\text { Curvularia } \\
\text { lunata }\end{array}$ & $\begin{array}{l}\text { Absidum } \\
\text { sp. }\end{array}$ \\
\hline$\overline{1}$ & $3^{\prime}$-Bromo-4'-(p-t.s.)C. & 25.0 & 8.0 & 7.0 & - & & 8.0 & 8.0 & 9.0 & 8.0 & . \\
\hline 2. & $\begin{array}{l}\text { 2-Hydroxy-3'-bromo- } \\
\text { 4'-(p-t.s.)C. }\end{array}$ & 18.0 & - & 12.0 & - & 11 & 8.0 & 9.5 & 9.0 & 7.0 & - \\
\hline 3. & $\begin{array}{l}\text { 2,4-Dihydroxy-3'-bromo- } \\
\text { 4'-(p-t.s.)C. }\end{array}$ & 10.0 & 7.0 & 15.0 & - & - & 13.0 & 18.0 & 10.5 & - & - \\
\hline 4. & $\begin{array}{l}\text { 2-Methoxy-3'-bromo- } \\
4^{\prime}(\text { p-t.s.)C. }\end{array}$ & 9.5 & - & 7.0 & - & 7 & - & 8.5 & 8.5 & 9.0 & - \\
\hline 5. & $\begin{array}{l}\text { 4-Methoxy-3'-bromo- } \\
\text { 4'(p-t.s.)C. }\end{array}$ & 8.0 & 8.0 & 12.0 & - & 12 & 9.0 & 8.0 & 14.0 & - & - \\
\hline 6. & $\begin{array}{l}\text { 3-Methoxy-4-hydroxy-3' } \\
\text { bromo-4'-(p-t.s.). }\end{array}$ & 8.5 & 11.0 & 8.0 & - & 7 & - & 8.0 & 9.0 & 10.0 & - \\
\hline 7. & $\begin{array}{l}\text { 3-Methoxy-4-hydroxy- } \\
\text { 3',5-dibromo-4'-(p-t.s.)C. }\end{array}$ & 10.0 & 8.0 & 7.0 & - & 8 & 13.0 & 10.5 & 15.0 & - & - \\
\hline 8. & $\begin{array}{l}\text { 3,4-Dimethoxy-3',5- } \\
\text { dibromo-4'-(p-t.s.)C. }\end{array}$ & 10.0 & 8.0 & 15.0 & - & - & 19.0 & 10.0 & 16.5 & 8.0 & - \\
\hline 9. & $\begin{array}{l}\text { 3-Nitro-3'-bromo- } \\
\text { 4'-(p-t.s.)C. }\end{array}$ & 8.0 & - & - & - & - & 9.0 & 8.0 & 7.0 & - & - \\
\hline 10. & $\begin{array}{l}\text { 4-Nitro-3'-bromo- } \\
\text { 4'-(p-t.s.)C. }\end{array}$ & 8.0 & - & 12.0 & - & - & - & 25.0 & 17.5 & - & - \\
\hline & $\begin{array}{l}\text { 4-Chloro-3'-bromo- } \\
\text { 4'-(p-t.s.)C. }\end{array}$ & 10.0 & 8.0 & 9.0 & 8.0 & 8 & 10.0 & 8.0 & 10.0 & 9.0 & 10.0 \\
\hline & Control Gresiofulvin & 23.0 & 24.5 & 23.0 & 23.0 & 23.0 & 27.0 & 25.0 & 25.0 & 23.0 & 25.0 \\
\hline
\end{tabular}

Here, (p-t.s.)C. denotes (p-tolyl sulphonamido) chalcone. (-) denotes no activity. 
Table III

Zones of inhibition in $\mathrm{mm}$

Name of the organism tested

\begin{tabular}{|c|c|c|c|c|c|c|c|c|c|c|}
\hline S.No. & $\begin{array}{l}\text { Names of the } \\
\text { compound }\end{array}$ & $\begin{array}{l}\text { Escheria- } \\
\text { chia coli }\end{array}$ & $\begin{array}{l}\text { Lactobacillius } \\
\text { sp }\end{array}$ & $\begin{array}{l}\text { Pseudomonas } \\
\text { putina }\end{array}$ & $\begin{array}{l}\text { Rhizobium } \\
\text { japanicum }\end{array}$ & $\begin{array}{l}\text { Rhizobium- } \\
\text { lequminosa }\end{array}$ & $\begin{array}{l}\text { Salmonella } \\
\text { typhi }\end{array}$ & $\begin{array}{l}\text { Serratia } \\
\text { bachoria }\end{array}$ & $\begin{array}{l}\text { Staphylococcus } \\
\text { aureus }\end{array}$ & $\begin{array}{l}\text { Is Vibrio- } \\
\text { cholarae }\end{array}$ \\
\hline 1. & 3'-Bromo-4'(p-t.s.)C. & _ & 9.0 & 9.0 & 8.0 & - & - & - & - & 8.0 \\
\hline 2. & $\begin{array}{l}\text { 4-Chloro-3'-bromo-4' } \\
\text { (p-t.s.)C. }\end{array}$ & 11.5 & 10.5 & 10.5 & 8.0 & - & - & - & - & 10.5 \\
\hline 3.2 & $\begin{array}{l}\text { 2-Hydroxy-3'-bromo-4' } \\
\text { (p-t.s.)C. }\end{array}$ & - & 8.0 & 8.0 & 8.0 & - & - & - & - & - \\
\hline 4. & $\begin{array}{l}\text { 2,4-Dihydroxy-3'-bromo- } \\
\text { (p-t.s.)C. }\end{array}$ & - & 11.0 & 22.0 & 10.0 & - & - & - & - & 8.0 \\
\hline 5. & $\begin{array}{l}\text { 2-Methoxy-3'-bromo-4'- } \\
\text { (p-t.s.)C. }\end{array}$ & 8.0 & 8.0 & 9.0 & 9.0 & - & - & - & - & 10.0 \\
\hline 6. & $\begin{array}{l}\text { 4-Methoxy-4-hydroxy-3'- } \\
\text { (p-t.s.)C. }\end{array}$ & 8.0 & 21.0 & 16.0 & 10.0 & - & - & - & 8.0 & 10.0 \\
\hline 7. & $\begin{array}{l}\text { 3-Methoxy-4-hydroxy-3'- } \\
\text { (p-t.s.)C. }\end{array}$ & - & 8.0 & 9.0 & 8.0 & - & - & - & - & 10.0 \\
\hline 8. & $\begin{array}{l}\text { 3-Methoxy-4-hydroxy-3', } \\
\text { 5-dibromo-4'-(p-t.s.)C. }\end{array}$ & - & 9.5 & 9.0 & 10.0 & - & - & - & - & 9.0 \\
\hline 9. & $\begin{array}{l}\text { 3,4-Dimethoxy-3',5- } \\
\text { dibromo-4'-(p-t.s.)C. }\end{array}$ & - & 10.0 & - & - & - & - & - & - & 10.0 \\
\hline 10. & $\begin{array}{l}\text { 3-Nitro-3'-bromo-4'- } \\
\text { (p-t.s.)C. }\end{array}$ & - & 11.0 & 10.0 & 10.0 & 9.0 & - & - & - & 10.0 \\
\hline \multirow[t]{2}{*}{11.} & $\begin{array}{l}\text { 4-Nitro-3'-bromo-4'- } \\
\text { (p-t.s.)C. } \\
\text { Control }\end{array}$ & - & 9.0 & 16.0 & 9.5 & - & - & - & - & 10.0 \\
\hline & Streptomycin & 28.0 & 29.0 & 29.0 & 28.0 & 34.0 & 43.5 & 28.0 & 35.0 & 35.0 \\
\hline
\end{tabular}

(p.t.s.)C. denotes - (p-toly1 sulphonomido) chalcone (-) denotes no activity. 
Acknowledgement :

The authors are thankful to the Heads, Department of Chemistry and Botany of Dr. H.S. Gour Vishwavidyalaya, Sagar for providing laboratory facilities and organisms.

\section{References}

1. Dandeganoker SH and Shelar A R, J. Shivaji Univ., 1971, 4(8), 77.

2. Real Laliberate, David Camhell and Gen. Brodorison, J. Pharm. Sci., 1947, 2(2), 37-43.

3. Misra S SJ. Indian Chem. Soc., 1973, 50, 68.

4. Mehta KJ and Parikh A R, J. Inst. Chemists (India), 1979, 51, 60-61.

5. $\quad(\mathrm{Km})$ Shah N J, (Km) Jhaveri LC and Naik H B, J. Indian Chem. Soc., 1981, 58, 300-302.

6. Joshi R S and Naik H B, J. Inst. Chemists (India), 1980, 52, 184-186.

7. Nayak A, Nayak P L, Sabata B K and Rout M K, J. Indian Chem, Soc.,1964, 41, 507-512.

8. (Miss) Trakroo P P and Mukhelkar A J, J. Indian Chem. Soc., 1964, 41, 595-599.

9. Maruzzella J C and Henry PA, J. Amer. Pharm. Assoc., 1958, 47, 471. 


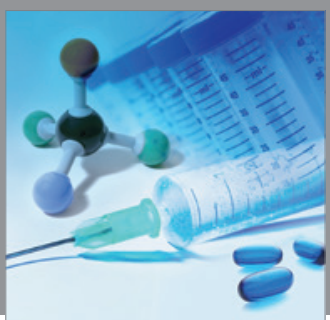

International Journal of

Medicinal Chemistry

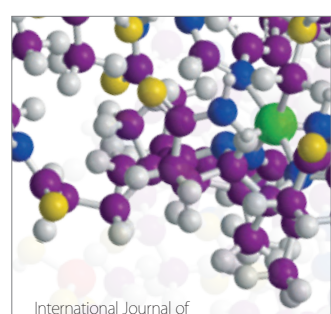

Carbohydrate Chemistry

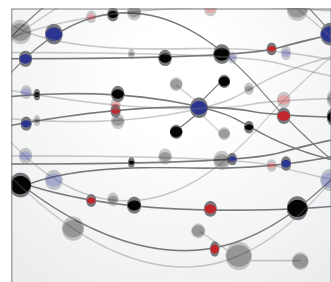

The Scientific World Journal
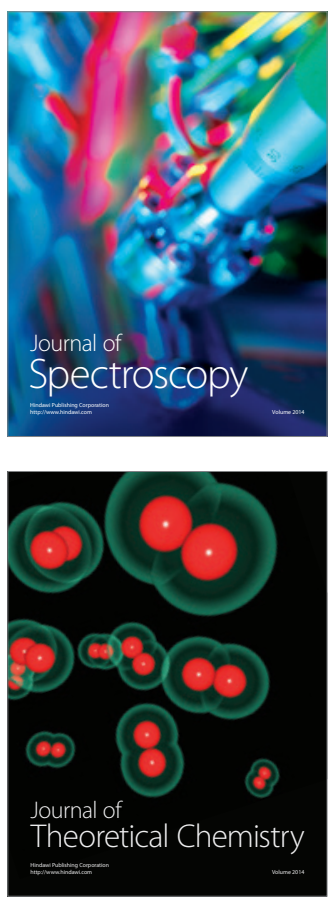
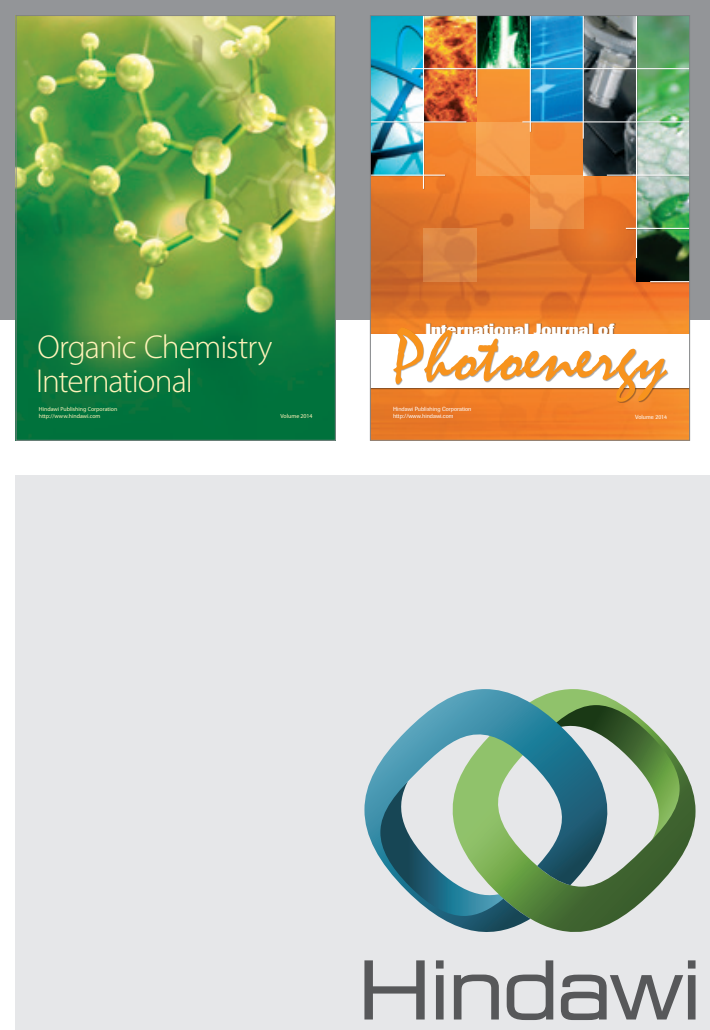

Submit your manuscripts at

http://www.hindawi.com
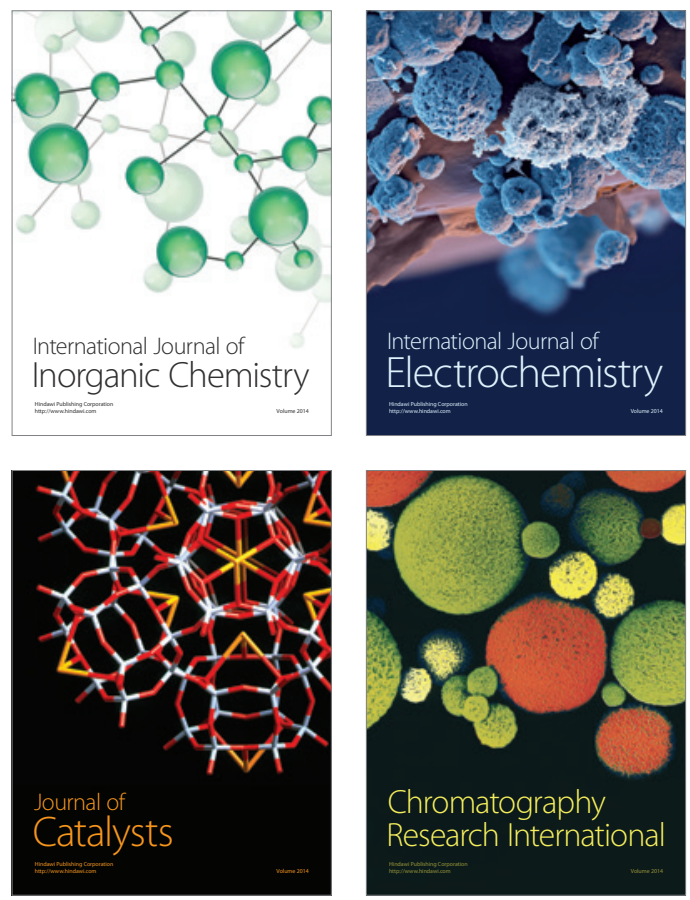
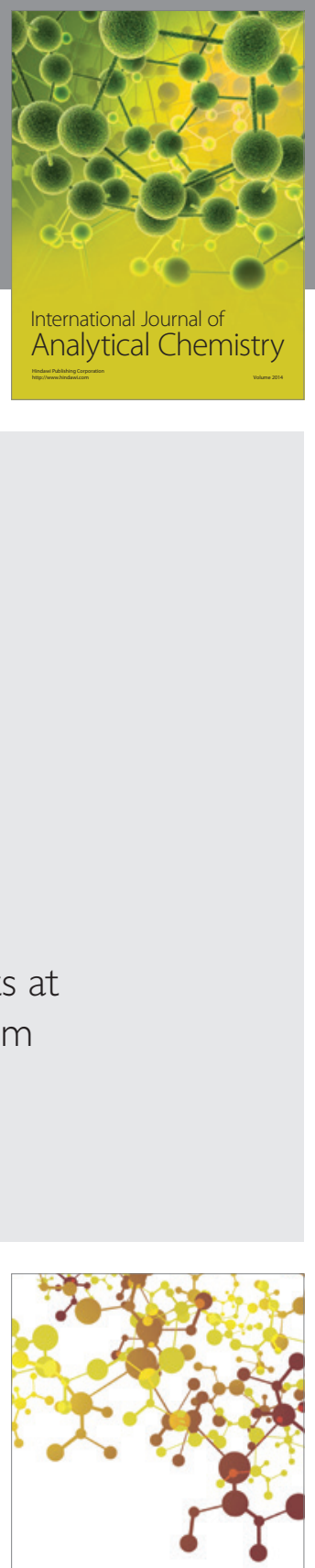

Journal of

Applied Chemistry
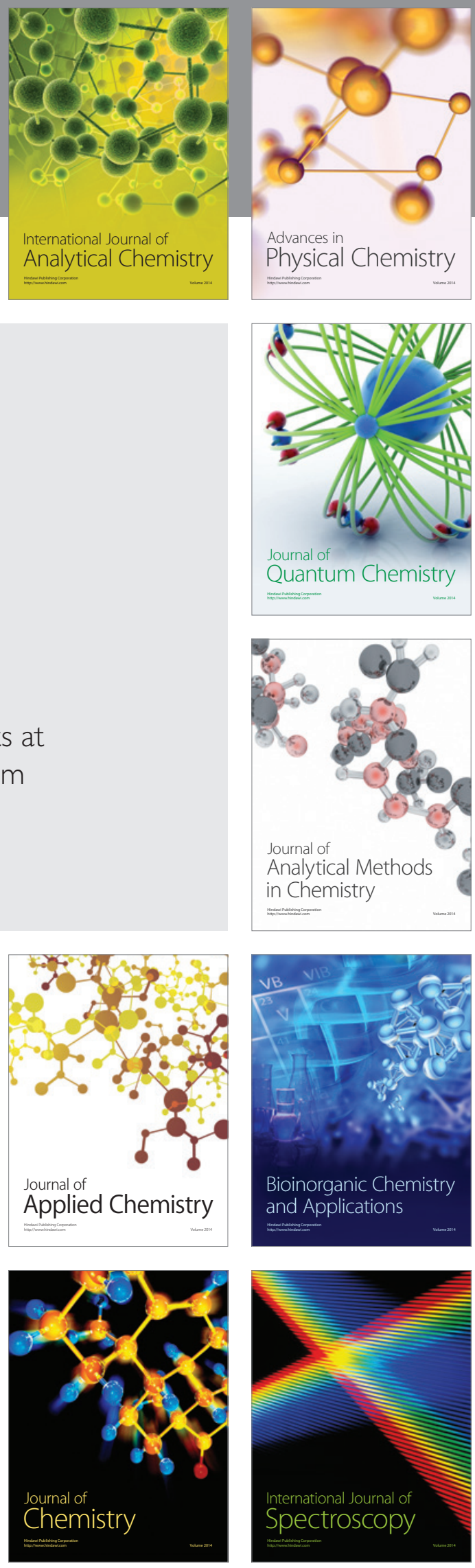